Scientific Journal of Hamadan Nursing \& Midwifery Faculty - ISSN 2008-2819

\title{
Investigating the Relationship Between the Meaning of Life and Self-Esteem with the Quality of Life of the Elderly Living in the Home and Living in the Nursing Home
}

\author{
Ezatolah Ghadampour ${ }^{1}$, Leila Heidaryani ${ }^{2}$, Farnaz Radmehr ${ }^{3 *}$
}

1. Associate Professor, Department of Psychology, Faculty of Literature and Human Sciences, Lorestan University, Khorramabad, Iran

2. Ph.D. Student, Department of Psychology, Faculty of Literature \& Humanities, Lorestan University, Khorramabad, Iran

3. Ph.D. Student, Department of Psychology, Faculty of Social Sciences, Razi University, Kermanshah, Iran

\section{Article Info \\ Received: $\quad$ 2017/08/16 \\ Accepted: $\quad 2017 / 10 / 3$ \\ Published Online: 2017/10/25}

DOI:

10.30699/sjhnmf.26.a5.315

Original Article

Use your device to scan and read the article online

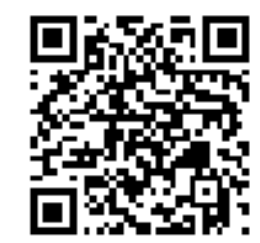

\section{Abstract}

Introduction: Elderly is a very important period of life in which many chronic illnesses threat the person well-being, also endangers their quality of life. This study aimed to compare the association of meaning in life and self-esteem with quality of life in elderly living at home and those in nursing home.

Methods: This was a descriptive-analytical study performed on 120 elderly women and men living in Khorramabad city between 2016 and 2017, including 60 elderly living at home and 60 elderly living in nursing homes. Subjects were selected by systematic random sampling) Based on file number). To collect data, the Steger et al meaning in life (2006), Rosenberg Self-Esteem (1965) and Sherbvn \& Weir quality of life (1992) questionnaires were used. Data was analyzed using Pearson correlation test, multiple regression analysis with stepwise method and T-test.

Results: There was a significant difference between meaning in life ( $\mathrm{t}=3.63$, $P<0.01)$, self-esteem $(\mathrm{t}=3,69, P<0.001)$ and quality of life $(\mathrm{t}=4.05, P<0.001)$ between elderly living at home and those living in nursing homeMultiple stepwise regression analysis showed that meaning of life and self-esteem predicted $26 \%$ of quality of life variance.

Conclusion: It seems necessary to improve the quality of life of elderly by planning and programming appropriate policies, especially those living in nursing homes.

Keywords: Meaning in life, Self-Esteem, Quality of life, Elderly

Copyright (C) 2018, Sci J Hamadan Nurs Midwifery Fac. This is an open-access article distributed under the terms of the Creative Commons Attribution-noncommercial 4.0 International License which permits copy and redistribute the material just in noncommercial usages, provided the original work is properly cited.

How to Cite This Article:

Ghadampour E, Heidaryani L, Radmehr F. Investigating the relationship between the meaning of life and self-esteem with the quality of life of the elderly living in the home and living in the nursing home. Sci J Hamadan Nurs Midwifery Fac. 2018; 26 (5): 315-322 


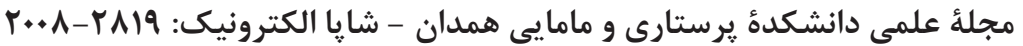

مقاله يخوهشى

بر رسى رابطؤ معناى زندگى و عزتنفس با كيفيت زندكى سالمندان ساكن منزل و مقيم سراى سالمندان

عزتاله قدميور'، ليلا حيدريانى '، فرناز رادمهر

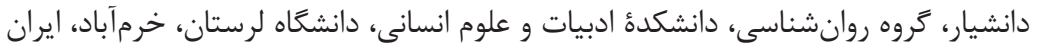

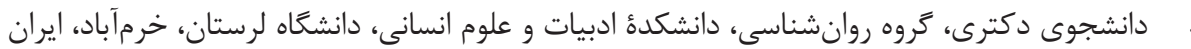

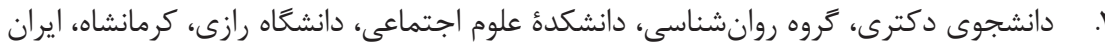

\begin{abstract}
קكيده

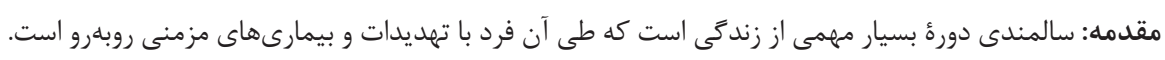

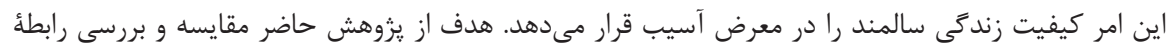

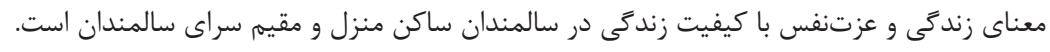

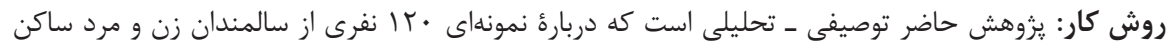

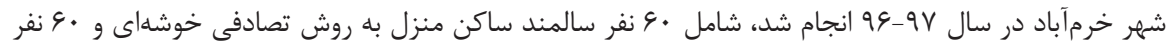

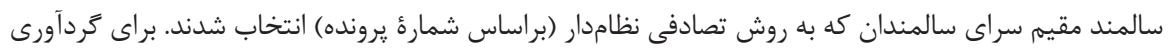

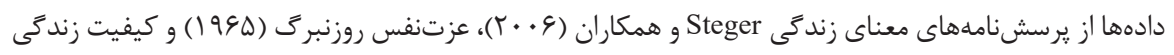

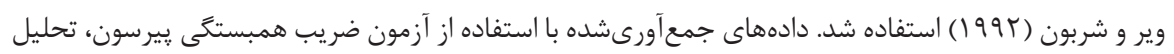
ركرسيون جند

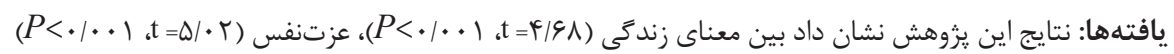

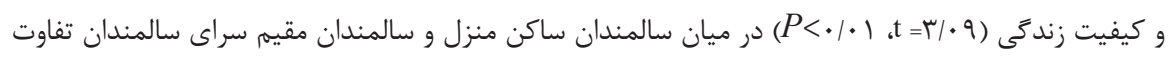

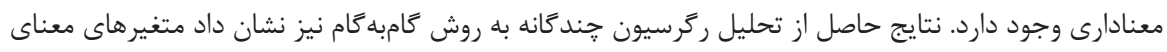

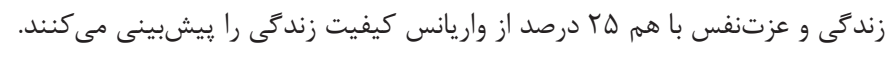

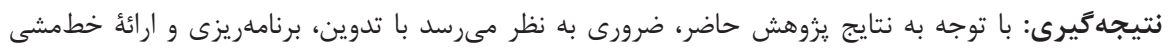

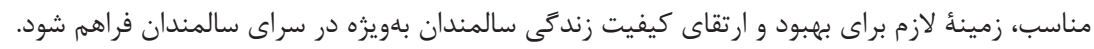

وازههاى كليدى: معناى زندگى، عزتنفس، كيفيت زندگى، سالمندان

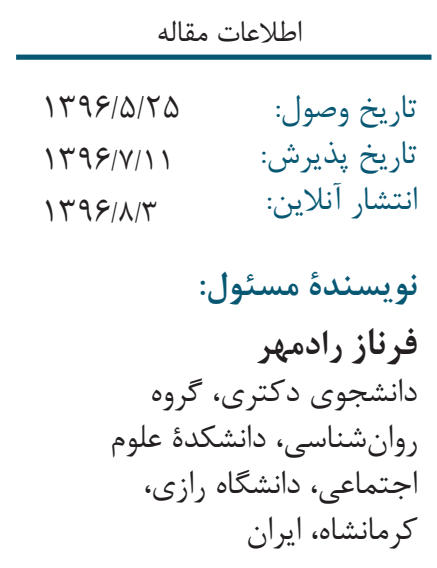

يست الكترونيك: radmehr.p12@gmail.com

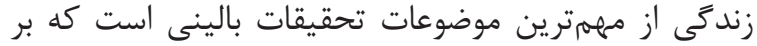

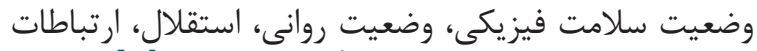

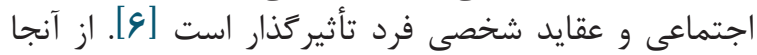

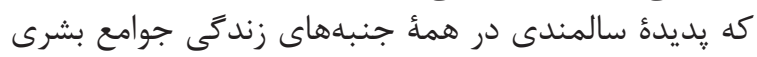

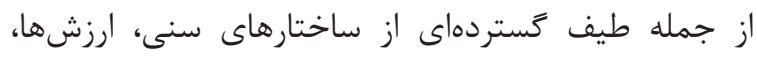

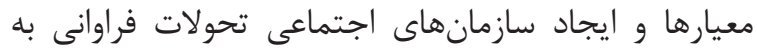

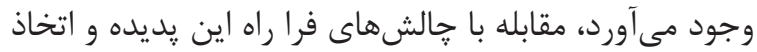

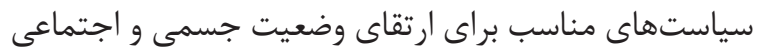

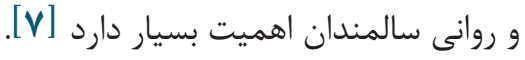
شناسايى عواملى كه بر سطح كيفيت زندگى تأثير زذار

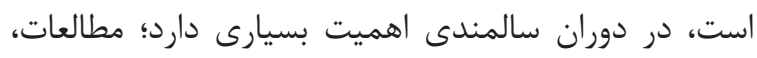

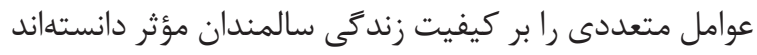

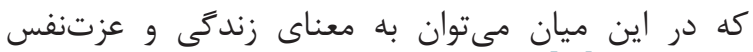

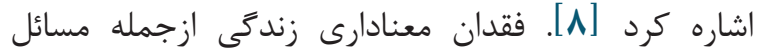

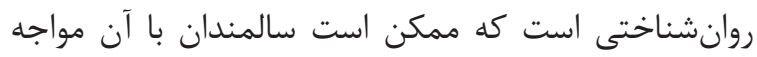
شوند. تغييرات جسمانى و مشكلات روحى - روانى در اواخر
سالمندى دورهاى از زندگى است كه طعى آن سالمندان در

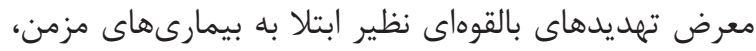

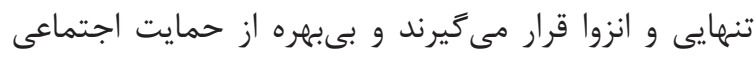

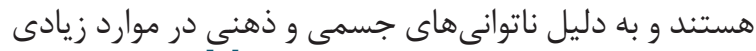

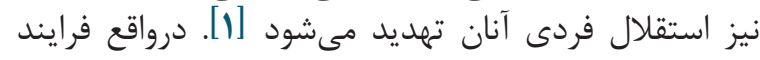

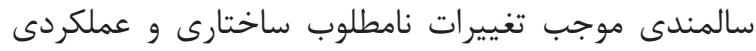

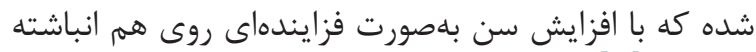

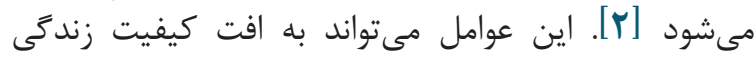

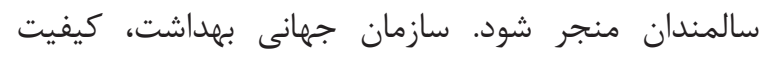

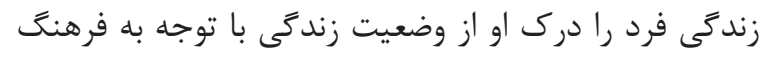

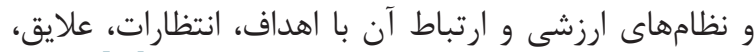

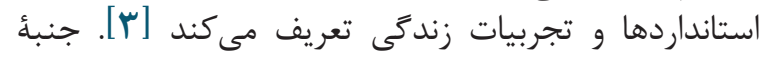

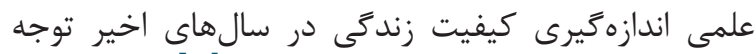

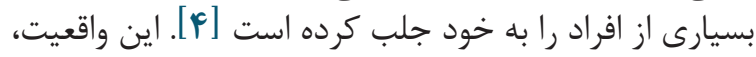

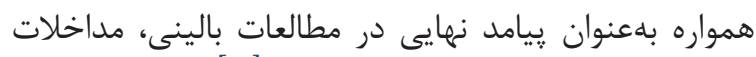

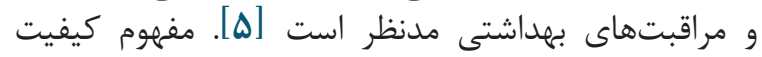


سويى نتايج متضادى كه در زمينأ محيط زندكى سالمندان

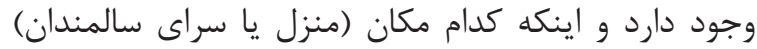

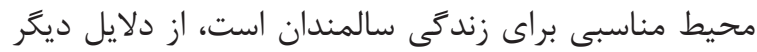

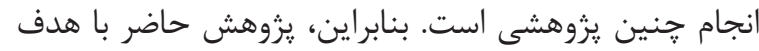

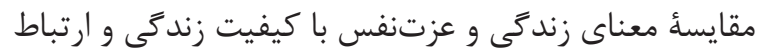

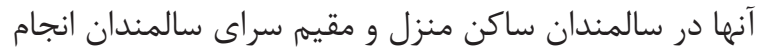

\section{روش كار}

روش يزوهش حاضر با توجه به ماهيت موضوع و اهداف

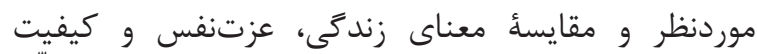

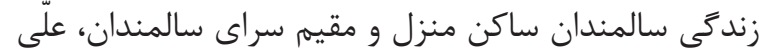

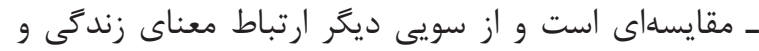

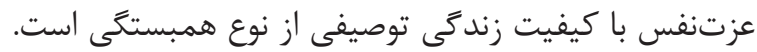

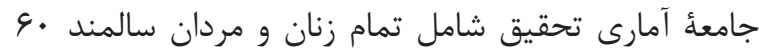

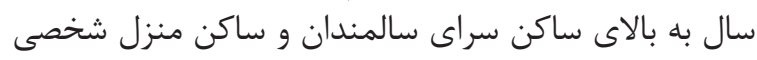

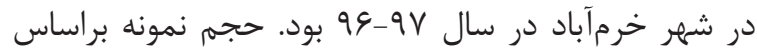

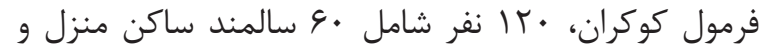

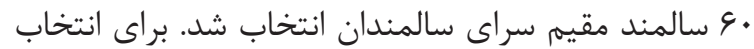

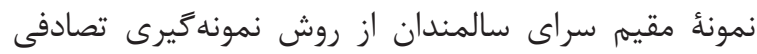

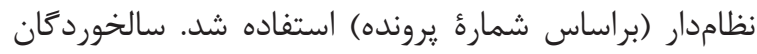

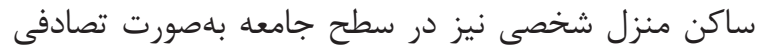

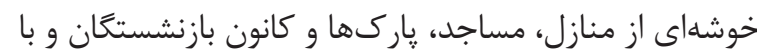

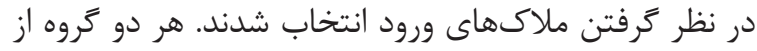
نظر سن و جنس با همديكر همتا شدند.

ملاكهاى ورود به يزوهش داشتن سن بيشتر از • • سال،

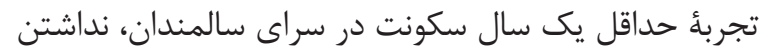

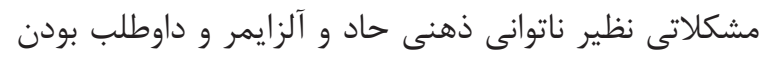

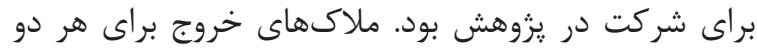

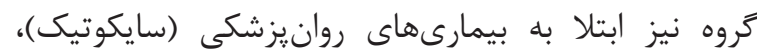

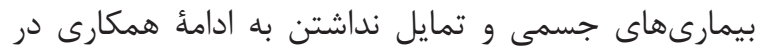

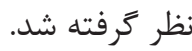

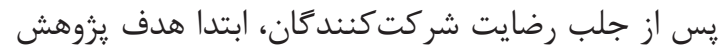

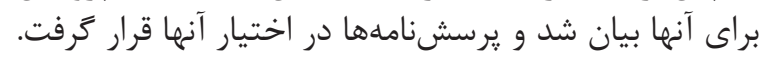

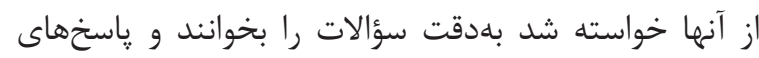

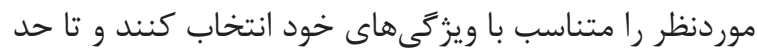

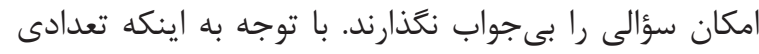

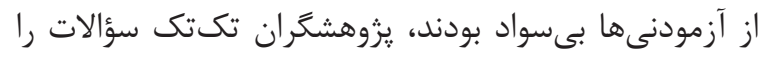

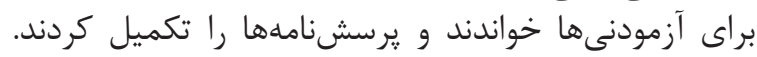

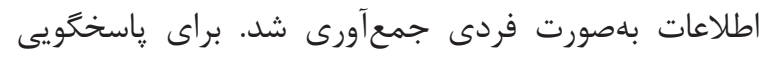

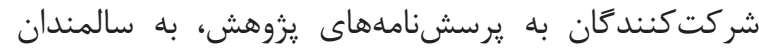

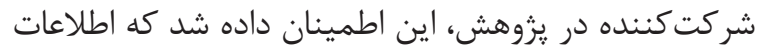

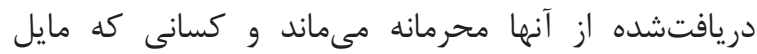

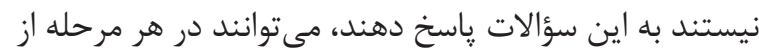
ياسخكويى، همكارى خود را إ ادامه ندهند.

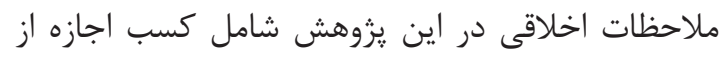

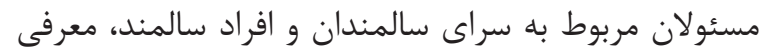
خود به واحدهاى بزوهش و تشريح اهداف و ماهيت يزولهش،

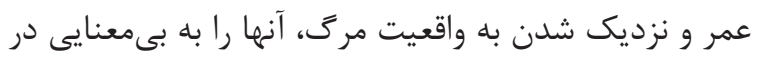

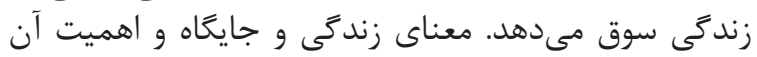

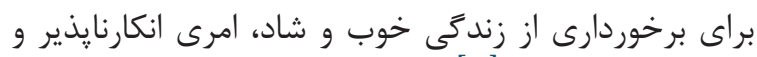

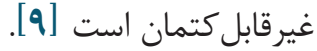

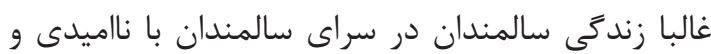

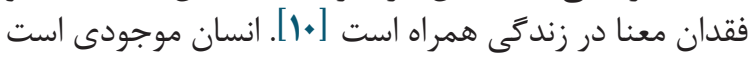

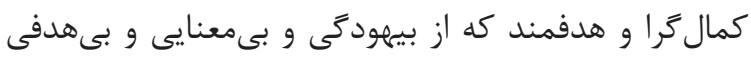

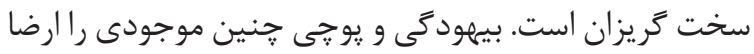

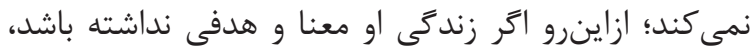

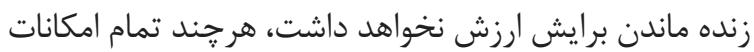

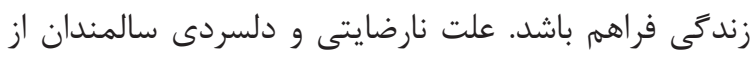

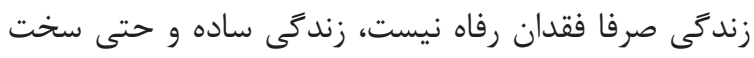

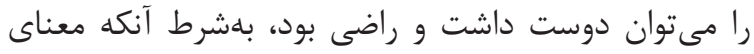

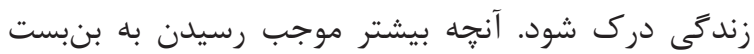

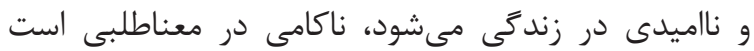

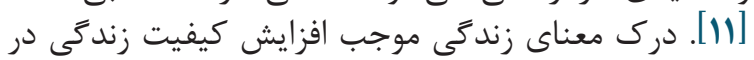

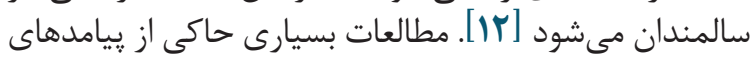

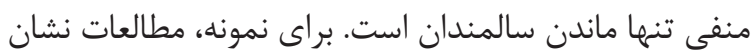

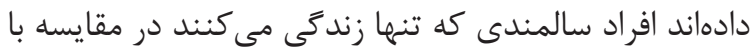

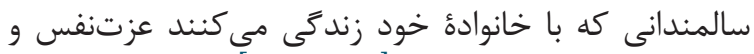

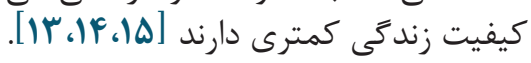

Pnina

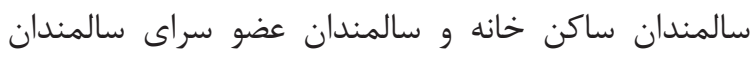

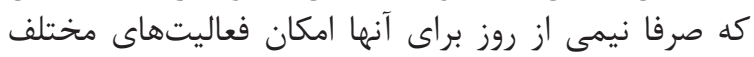

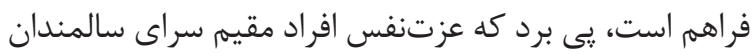

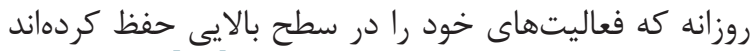

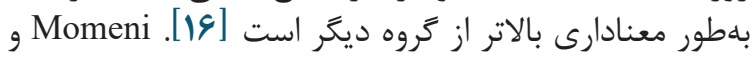

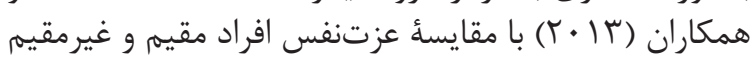

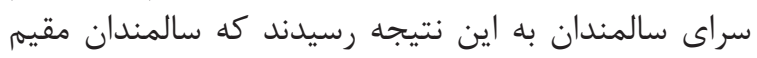

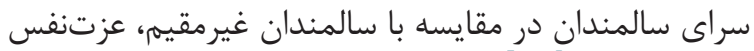

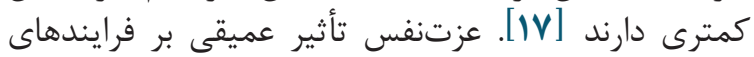

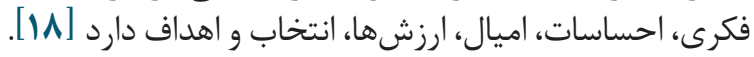

Zare

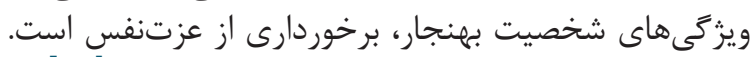

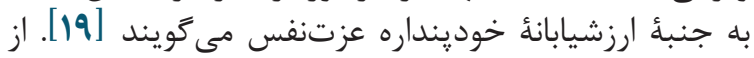

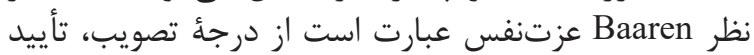

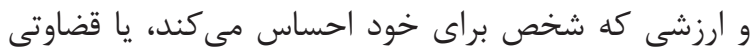

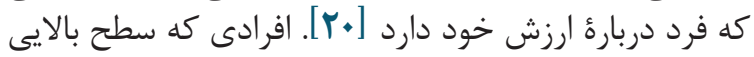

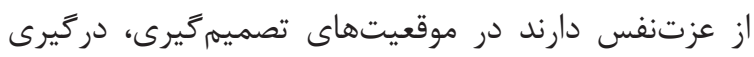

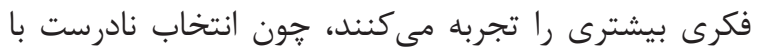

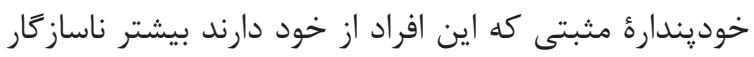

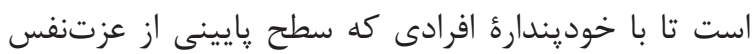

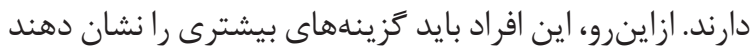

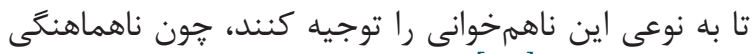

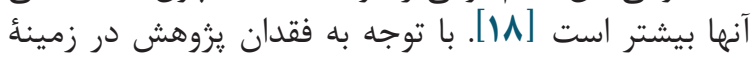

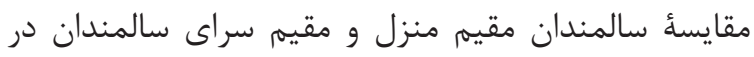

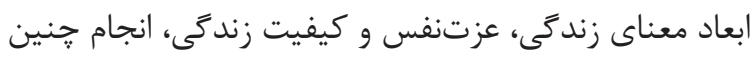

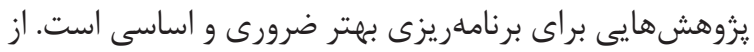




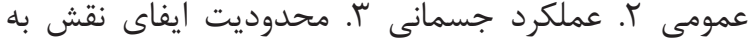

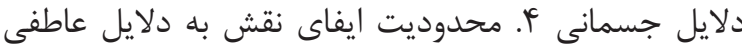

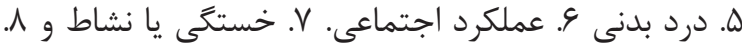

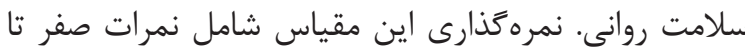

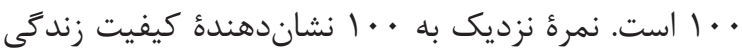

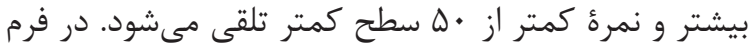

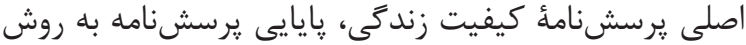

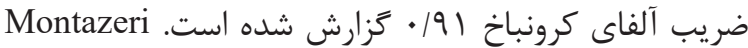

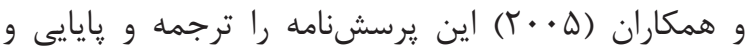

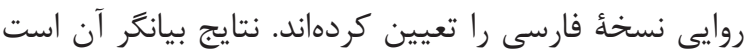

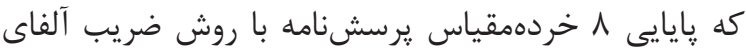

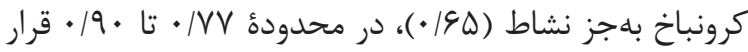

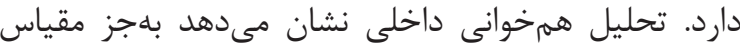

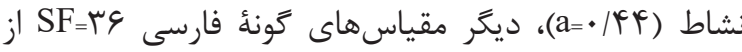

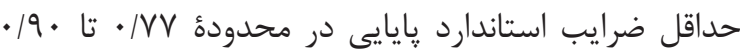

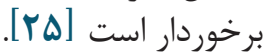

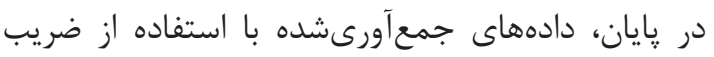

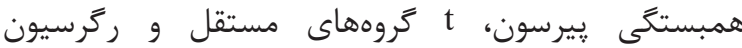

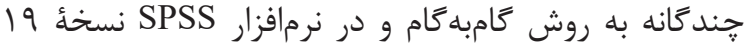

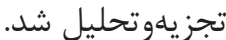

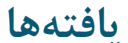

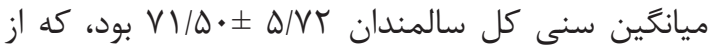

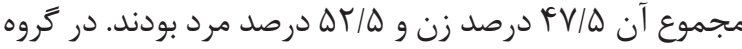

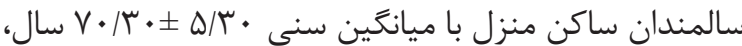

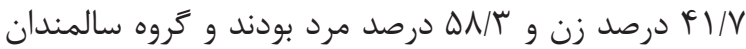

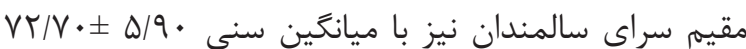

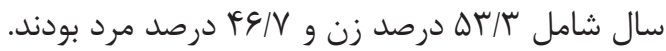
اطلاعات توصيفى (ميانكين و انحرافمعيار) متغيرهاى

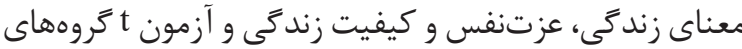

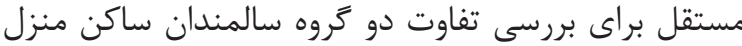

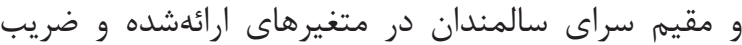

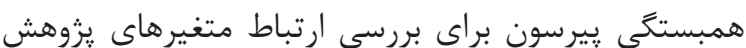

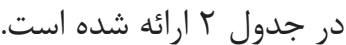

كسب رضايت از سالمندان و جلب مشاركت آنان در يزوهش آنش

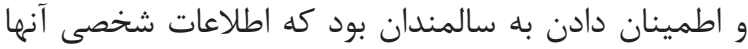

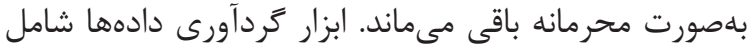

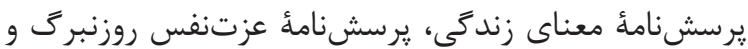

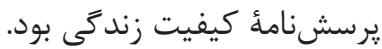

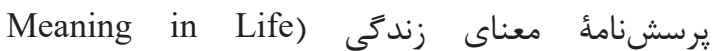
Questionnaire

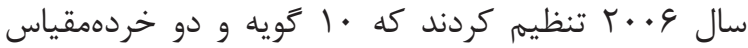

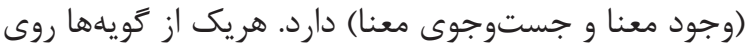

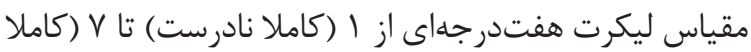

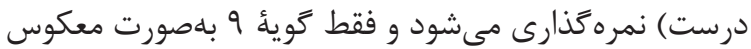

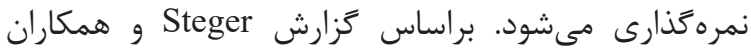

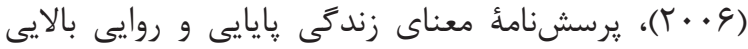

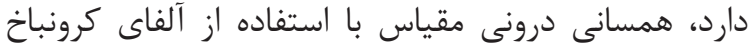

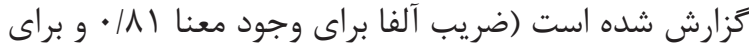

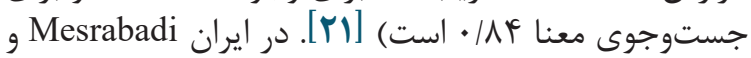

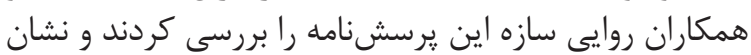

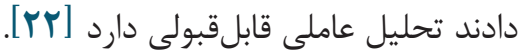

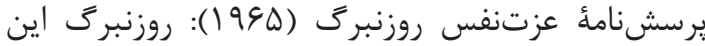

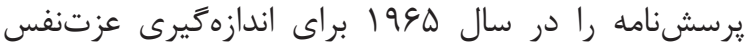

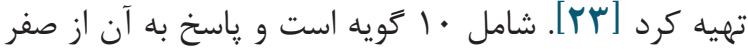

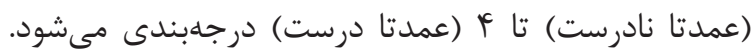

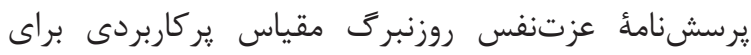
ارزيابى عزتنفس است كه بايايى بازازمايى آزمون

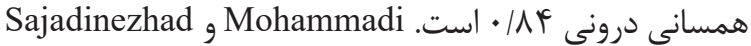

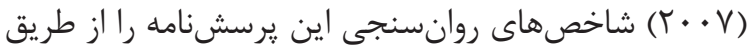

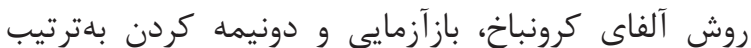

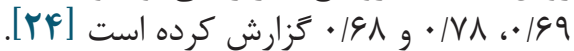

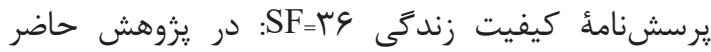

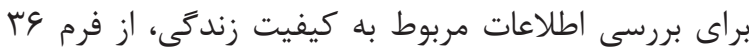

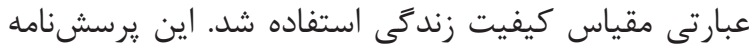

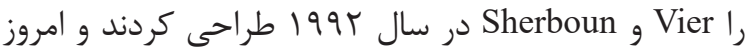

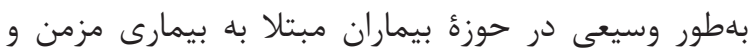

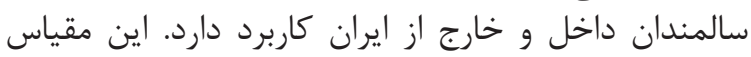

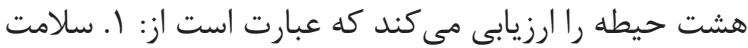

جدول 1. اطلاعات جمعيتشناختى واحدهاى يزوهش

\begin{tabular}{|c|c|c|c|c|c|}
\hline \multicolumn{2}{|c|}{ سالمندان مقيم سراى سالمندان } & \multicolumn{2}{|c|}{ سالمندان ساكن منزل } & \multirow{2}{*}{ غير ل غير } & \\
\hline درصد | لمد & 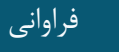 & درصد | د | لمد & 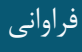 & & \\
\hline r $9 / \mathrm{V}$ & 19 & $F \& / V$ & ru & Y. & \\
\hline 4. & rq & $\Delta \cdot$ & $r$. & 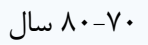 & 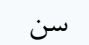 \\
\hline $1 \% / \pi$ & $\wedge$ & $r / \pi$ & r & • • 9 سال & \\
\hline$\Delta r / T$ & rt & fi/v & ra & زن & \\
\hline$\varphi 9 / \mathrm{V}$ & ru & N & ra & مرد & ي \\
\hline
\end{tabular}


جدول r. مقايسٔ معناى زندكى، عزتنفس و كيفيت زندكى در دو كروه سالمندان ساكن

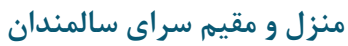

\begin{tabular}{|c|c|c|c|c|c|c|c|}
\hline سطح معنادارى & $\mathbf{t}$ & df & انحرافمعيار & ميانكين & 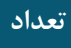 & 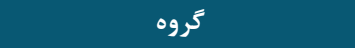 & متغير ها \\
\hline \multirow{2}{*}{$\cdot 1 \cdot \cdot 1$} & \multirow{2}{*}{$r / 9 \Lambda$} & \multirow{2}{*}{111} & $9 / 94$ & $9 \cdot / V 4$ & 4. & سالمندان ساكن منزل & \multirow{2}{*}{ معناى زندگى } \\
\hline & & & $N \cdot F$ & $\Delta G / T V$ & 4. & سالمندان مقيم سراى سالمندان & \\
\hline \multirow{2}{*}{$\cdot / \cdot 1$} & \multirow{2}{*}{$\Delta / \cdot r$} & \multirow{2}{*}{111} & $r / 4 G$ & $r \cdot / 4 t$ & 4. & سالمندان ساكن منزل & \multirow{2}{*}{ عزتنفس } \\
\hline & & & $T / T)$ & TN/AS & 4. & سالمندان مقيم سراى سالمندان & \\
\hline \multirow{2}{*}{$\cdot 1 \cdot 1$} & \multirow{2}{*}{$r / \cdot 9$} & \multirow{2}{*}{111} & $V / \Delta \Delta$ & $V \Delta / \cdot \Delta$ & 4. & سالمندان ساكن منزل & \multirow{2}{*}{ كيفيت زندگى } \\
\hline & & & V/qu & $Q V / D V$ & 4. & سالمندان مقيم سراى سالمندان & \\
\hline
\end{tabular}

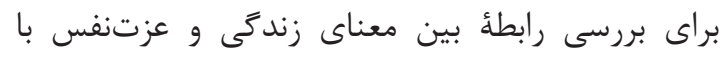

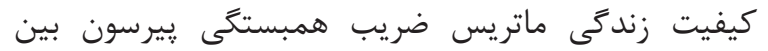

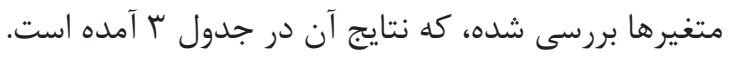

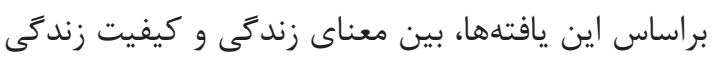

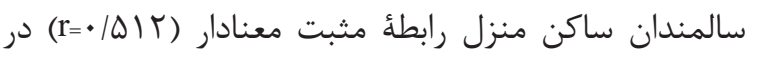

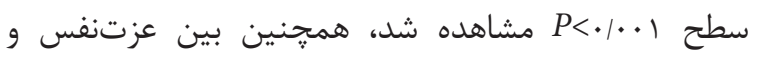

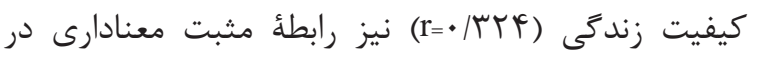
سطح

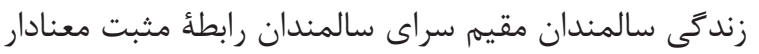
(r=•/TFA)

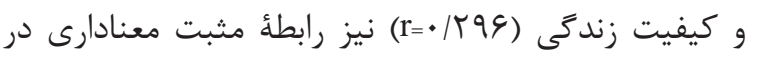

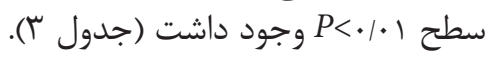

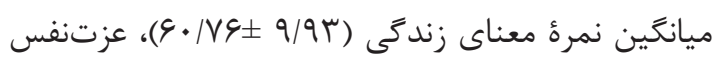
ساكن

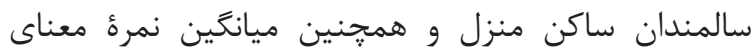

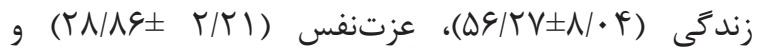
كيفيت زندگى (

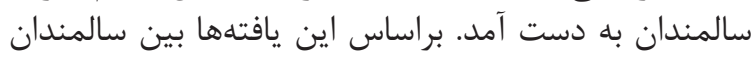

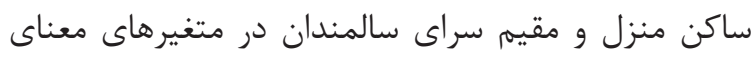

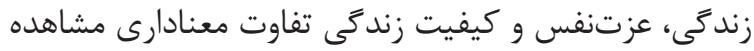

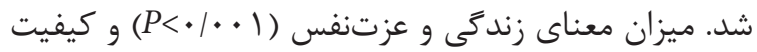

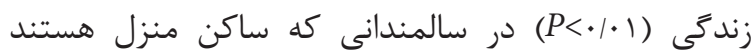

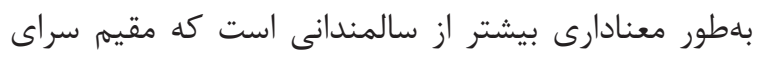

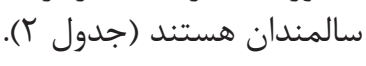
جدول ץ. همبستگى معناى زندگى و عزتنفس با كيفيت زندگى در دو گروه سالمندان ساكن منزل و مقيم سراى سالمندان

\begin{tabular}{|c|c|c|c|c|}
\hline \multicolumn{2}{|l|}{ كيفيت زندكى } & معناى زندَى & متغير ها & \\
\hline & & 1 & معناى زندكى & \multirow{4}{*}{ سالمندان ساكن منزل } \\
\hline & 1 & $\cdot|r q|$ & عزتنفس & \\
\hline 1 & . THY" & $\cdot|\Delta| Y^{* * a n}$ & كيفيت زندكى & \\
\hline \multirow[t]{3}{*}{$P<\cdot 1 \cdot \cdot 1$} & $P<\cdot / \cdot 1$ & & & \\
\hline & & 1 & معناى زندَّى & \multirow{4}{*}{ سالمندان مقيم سراى سالمندان } \\
\hline & 1 & . $|r M| *$ & عزتنفس & \\
\hline 1 & ./ $99^{*}$ & $\cdot / M Y \Lambda^{*}$ & كيفيت زندگى & \\
\hline${ }^{*} P<\cdot 1 \cdot \cdot 1$ & & & & \\
\hline
\end{tabular}

جدول F. نتايج ضرايب مدل ركرسيونى معناى زندَّى و عزتنفس بر كيفيت زندكى

\begin{tabular}{|c|c|c|c|c|c|c|c|}
\hline \multirow{2}{*}{$\mathbf{R}^{2}$} & \multirow{2}{*}{$\mathbf{R}$} & \multirow{2}{*}{ سطح معنادارى } & \multirow{2}{*}{$\mathbf{t}$} & \multirow{2}{*}{ ضرايب استاندارد } & \multicolumn{2}{|c|}{ ضرايب غيراستاندارد } & \\
\hline & & & & & خطاى استاندارد & B & \\
\hline \multirow{2}{*}{$\cdot / r \Delta \Delta$} & \multirow{2}{*}{$\cdot 10 \cdot \Delta$} & $\cdot 1 \cdot \cdot 1$ & $F / q F V$ &.$/ F T$. & $\cdot / 1 \cdot 1$ & س سه/. & معناى زندكى \\
\hline & & $\cdot / \cdot \Delta$ & $r / \cdot r q$ &.$/ 1 \mathrm{Vr}$ & $\cdot \mid \cdot \wedge 1$ & .1190 & عزتنفس \\
\hline
\end{tabular}




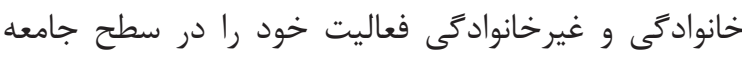

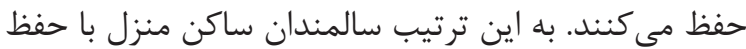

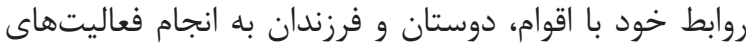

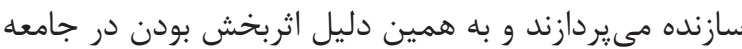

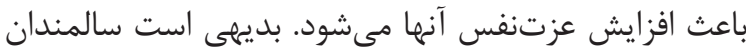

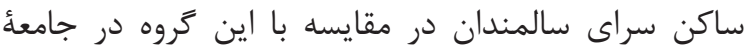

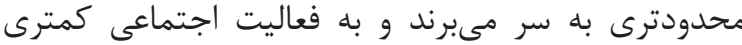

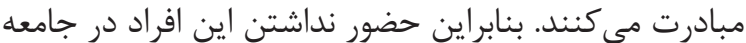

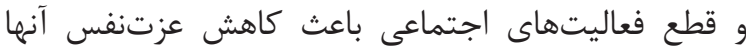

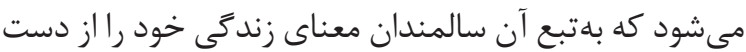

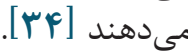

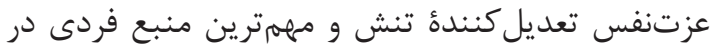

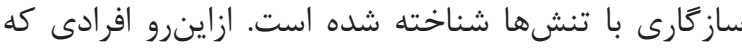

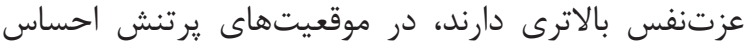

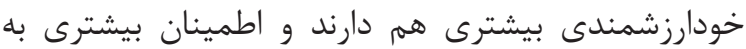

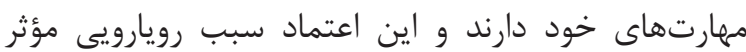

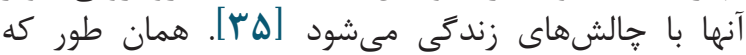

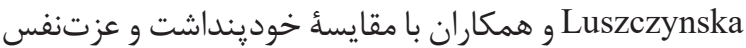

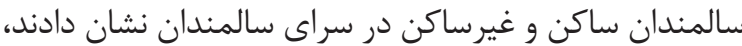

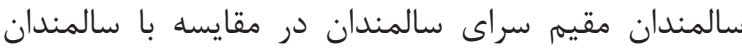

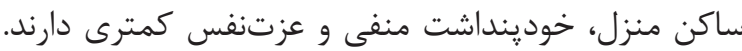

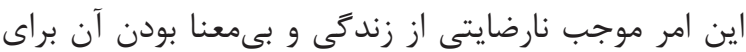

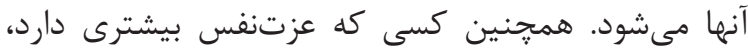

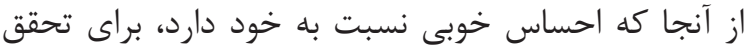

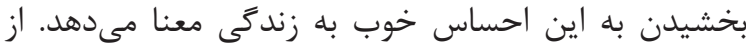

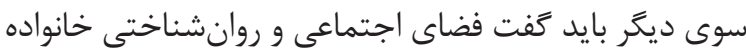

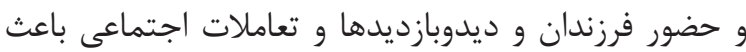

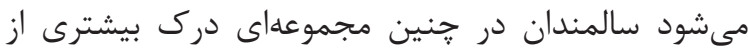

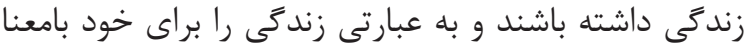

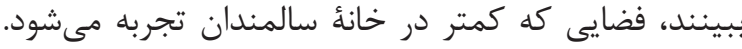

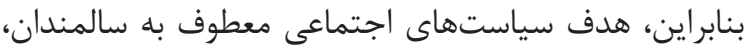

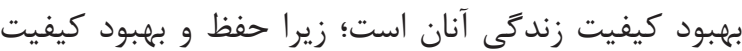

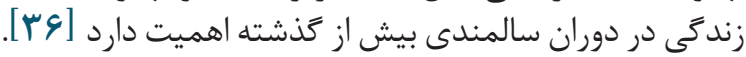

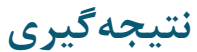

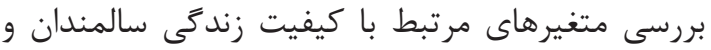

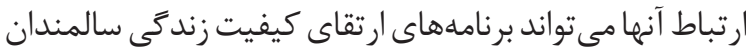

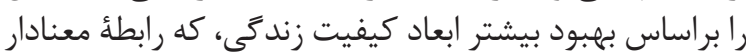

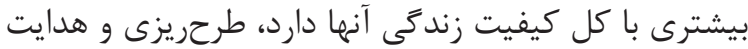

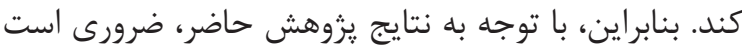

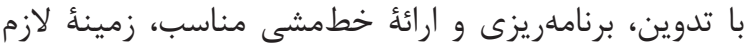

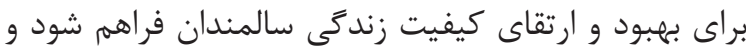

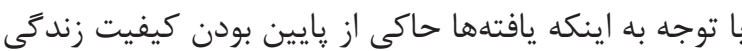

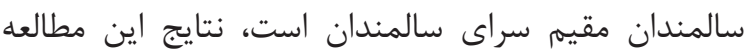

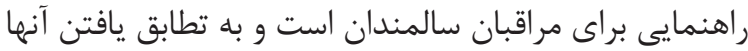

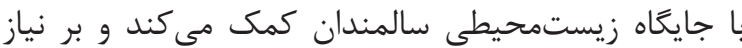

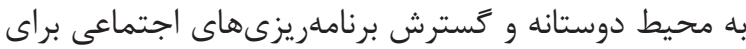

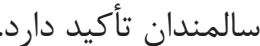

براساس نتايج ضرايب بتاى كزارش زشده، هر دو مت متغير

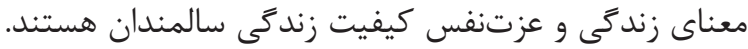

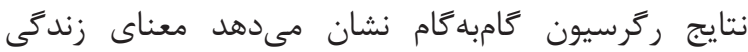

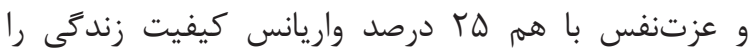

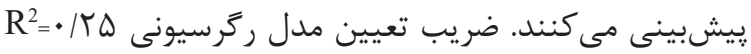

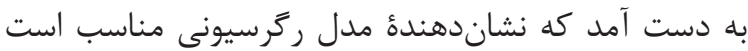

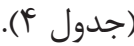

\section{بحث}

هدف از يزوهش حاضر مقايسه و بررسى رابطؤ معناى

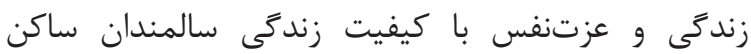

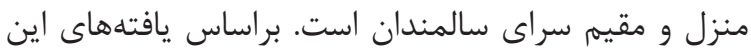

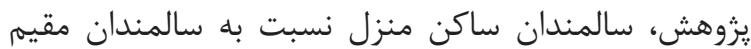

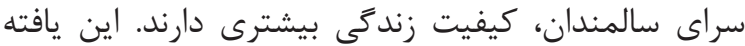

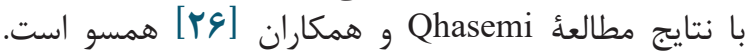

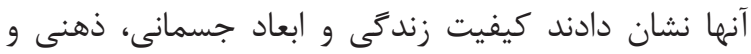

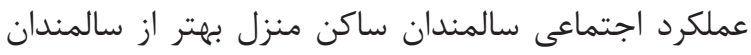

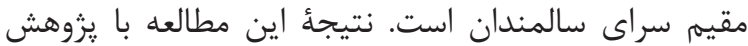
TYumin

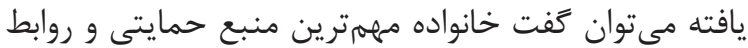

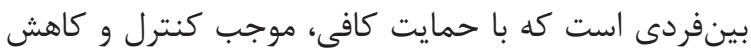

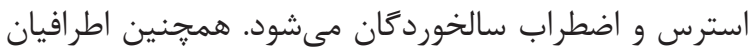

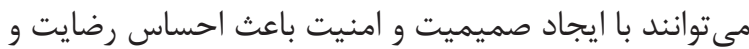
افزايش كيفيت زندكى شوند [rᄉ]

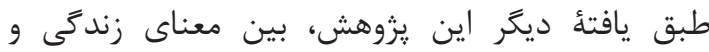

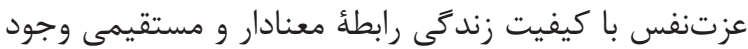

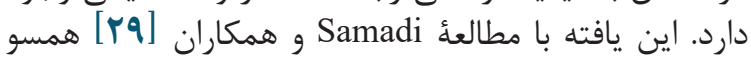

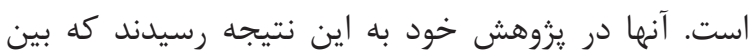
كيفيت زندگى و عزتنفس ارتباط وجود دارد. Soheilizadeh

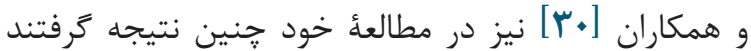

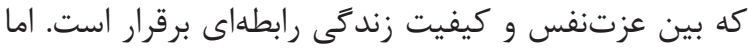

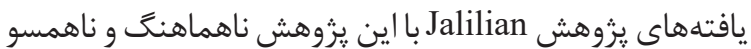

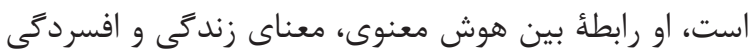

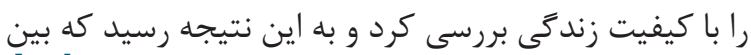

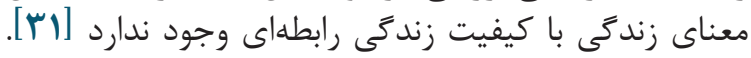

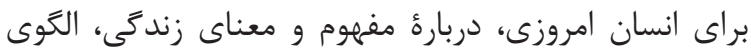

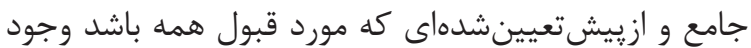

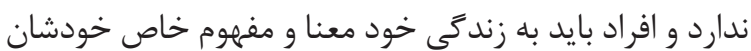

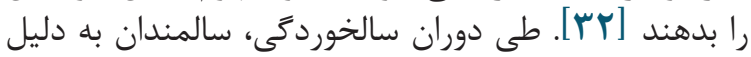

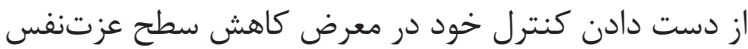

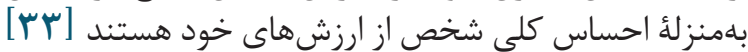

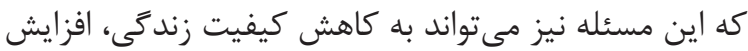
افسردىى و ساير بيمارىهاى جسمى ونى و... منجر شود.

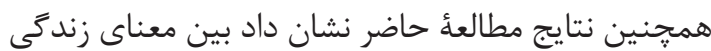

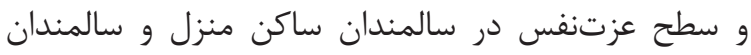

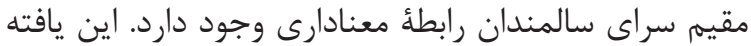

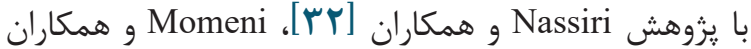

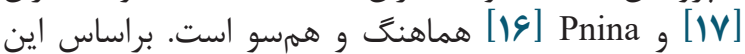
يافته سالمندانى كه ساكن منزل هستند با ايفاى نقشهاى الثراي 


$$
\begin{aligned}
& \text { يروانه رادمهر و همكاران إن } \\
& \text { اخلاق در يروهش توجه داشته و با شماره } \\
& \text { دانشخاه لرستان است. } \\
& \text { تعارض منافع } \\
& \text { در اين مطالعه تضاد منافع وجود ندارد. }
\end{aligned}
$$

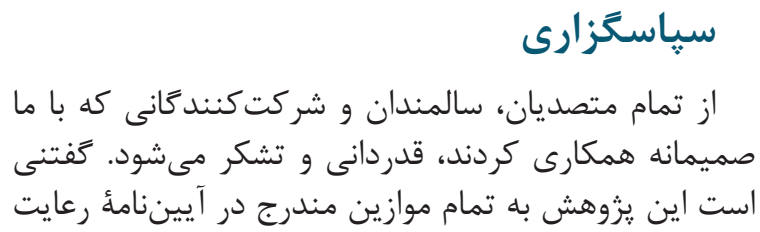

\section{References}

1. Canbaz S, Tevpik- Senter A, Dabak S, Peksen Y. The prevalence of chronic diseases and quality of life in elderly people in Samsun. Turk J Scio. 2003; 33: 335- 40.

2. Pourabdel S, Abbasi M. Pirani D, Abbasi M. The relationship between life expectancy and psychological well-being with the quality of life of the elderly. J of Aging Psych. 2015; 1(1): 57-65.

3. Zarghami M, Khademlou M, SheikhMonsey F, Behroozi B. Study of satisfaction status of quality of life in medical students and natural resources. J of Mazan Uni of Medi Scie. 2012; 22(89): 42-48.

4. Theodoropoulou S, Leotsakou C, Baltathakis I. Quality of life and psychology of 53 long-term survivors of allergenic bone marrow transplantation-Hippocratic. 2006; 6(1): 19.

5. Fayers P.M, Machin D. Quality of life: Assessment Analysis, and Interpretation. New York: Wiely publisher. 2000: 25- 30. https://doi. org/10.1002/0470846283

6. Cadena J.M, Vinaccias S.M, Pcers A.P. The impact of disease on the quality of life, mental and family dysfunction in Colombian patients with rheumatoid. Arthritis. Journal of clinical Rheumatology. 2003; 9(3): 142- 50.https://doi. org/10.1097/01.RHU.0000073434.59752.f3 PMID:17041449

7. Rezvani M. Mansourian H, Ahmad Abadi H, Ahmadabadi F, ParvaeiHeredesht Sh. Measuring the Effective Factors on the Quality of Older Living in Rural Areas (Case Study: Neishabour City). J of Rural Stud. 2013; 4(2): 301-326.

8. Sari N, Ksayar E, Real S, Perfect h. Evaluation of quality of life and its effective factors in the elderly living in nursing homes of Mashhad- 1392. J Mazan Uni of Medi Scie. 2015; 23(1): 243-252.

9. King L.A, Hicks J.A, Krull J.L, Del Gaiso A.K. Positive affect and the experience of meaning in life. J of Perso and Soci Psych. 2006; 90(1): 17996.https://doi.org/10.1037/0022-3514.90.1.179 PMID: 16448317

10. Brody C.M, Seme 1 V.G. Strategies for therapy with the elderly: Living with hope and mean Ed. New York: Springer publishing company. 2005;
180.

11. Gholam Mohammadi H, Frooghan M, Bahrami F, Younesi, SJ.Fazi M. The Effectiveness of Religious Educational Content on the Significance of the Life of the Elderly Man in the Hospital. J of Disab Studi. 2013; 3(2): 34-42.

12. $\mathrm{Wu} \mathrm{CH}$, Yao G. Analysis of factorial invariance across gender in the Taiwan version of the Satisfaction with Life Scale. Personality and Individual Differences. 2006;40(6):1259-68. https://doi. org/10.1016/j.paid.2005.11.012

13. Sok SR, Yun EK. A comparison of physical health status, self-esteem, family support and health-promoting behaviours between aged living alone and living with family in Korea. J Clin Nurs. 2011;20(11-12):1606-12.https:// doi.org/10.1111/j.1365-2702.2010.03551.x PMID:21366741

14. Shin SH, Sok SR. A comparison of the factors influencing life satisfaction between Korean older people living with family and living alone. Int Nurs Rev. 2012;59(2):252-8.https:// doi.org/10.1111/j.1466-7657.2011.00946.x PMID:22591098

15. Liu N, Zeng L, Li Z, Wang J. Health-related quality of life and long-term care needs among elderly individuals living alone: a cross-sectional study in rural areas of Shaanxi Province, China. BMC Public Health. 2013;13:313. https://doi. org/10.1186/1471-2458-13-313 PMID:23566211 PMCID:PMC3642010

16. Pnina R. Self- esteem among elderly people receiving care insurance at home and at day centers for the elderly. J Interna Psych geriat. 2007; 19(6): 1097- 1109.

17. Momeni Kh, Karami J, Majzoubi, MR. Comparison of lifestyle, self-esteem and mental health of only elderly, non-nursing and elderly people. J of Develo Psych. 2013; 10(38): 139-148.

18. Pourhossein R, Sheikhan Sh, Gholamali Lavasani $M$. Investigating the effectiveness of self-review method on increasing happiness and self-respect in juvenile delinquents. J of Psych. 2014; 3(4): 61-72.

19. Zare N, Daneshahi F, Amini M, Razeghi M, Fal- 
lahzadeh MH. Relationship between academic status and general health and self-esteem in students of Shiraz University of Medical Sciences. Iranian Jof Medi Educ. 2007; 7(1): 59-67.

20. Baaren, BV. Theories on coping with loss: The impact of social support and self-esteem on adjustment to emotional and social loneliness following a partner's death in later life. J Geru. 2002, 57: 33-41. https://doi.org/10.1093/geron$\underline{\mathrm{b} / 57.1 . \mathrm{S} 33}$

21. Steger MF, Frazier P, Oishi S, Kaler M. The meaning in life questionnaire: Assessing the presence of and search for meaning in life. Journal of counseling psychology. 2006 Jan;53(1):80. https://doi.org/10.1037/0022-0167.53.1.80

22. Mesrabadi J, Ostwar N, Jafarian S. Structural and Diagnostic Validity of the Questionnaire of Meaning in Life in Students. J of Behav Scien. 2013; 7(1): 83-90.

23. Jahanit Tabesh A, TamnaeiFar Sh, Mohammad Khani P, Jokar M. Structured Clinical Interview for DSM-IV-TR Disorders, Tehran: Dangeh Publication. 2013.

24. Mohammadi NA, Sajadinezhad MS. The relationship among body image concern, fear of negative evaluation and self-esteem with social anxiety. Journal of Psychology, Tabriz University. 2007 2(5):55-70.

25. Montazeri A, Vahdaninia M, Goshtasebi A. Translation, Determination of Reliability and Validity of Farsi Persian Questionnaire $\mathrm{SF}=36$. J of Payesh. 2005; 5(1): 49-56.

26. Qhasemi H, Harirchi AM, Masnavi A, Rahgozar M, Akbarian M. Comparison of the quality of life of the elderly residents of the family and the residents of the elderly in Isfahan. J Soc welfa maga. 2010; 10(39): 177-200.

27. Yumin ET, Simsek TT, Sertel M, Ozturk A, Yumin M. The effect of functional mobility and balance on health-related quality of life (HRQoL) among elderly people living at home and those living in nursing home. Arch Gerontol Geriatr. 2011;52(3):e180-4. $\quad$ https://doi.org/10.1016/j. archger.2010.10.027 PMID:21167611

28. Farhadi A, Ebiyavi Z, Movahedi Y, Rahimi M, Mobasher M. Comparison and relationship between social support and stress with life satisfaction in the elderly in Khorramabad city in 2013. Lorestan University of Medical Sciences. 2013; 17(2): 14-22.

29. Samadi N, Safavi M, Mahmoudi M. Evaluation of quality of life and its relationship with self-esteem in type 2 diabetic patients referred to diabetes clinic of Ardabil Imam Khomeini Hospital in 2011: Short report. J of Rafsa Univ of Medi
Scie.2012; 12(3): 252-256.

30. Soheilizadeh M, Yahaghi Amjad A, Gholam Aliii, B. Relationship between self-esteem and resiliency with quality of life in patients with type 2 diabetes mellitus in Hamadan in 1394. J of Nutri Scien. 2016; 15(1): 1-8.

31. Jalilian A. The Relationship between Spiritual Intelligence, Meaning of Life and Depression with Quality of Life in Women with Breast Cancer in Kermanshah City in 2013-14, Master's thesis, Urmia University, Faculty of Literature and $\mathrm{Hu}$ manities. 2014.

32. Nassiri Z, Dadkhah A, KhodabakhshiKoulaei A. Comparison and comparison of happiness and self-esteem among the elderly living in boarding houses and elderly residents at home. J Elde quart. $2012 ; 7(2)$ : 18-25.

33. Wanjiru M, Gathogo J. The impact of gender on orphans self- esteem and academic performance among secondary school students in kirinyaga and nyeri counties of Kenya. Internatil resea $\mathrm{J}$ of arts and soci scie. 2014; 3(4): 98- 103.

34. Nakahara J. Effects of social activities outside the home on life satisfaction among elderly people living alone. Interna J of PsychStud. 2013; 5(1): 112- 120.https://doi.org/10.5539/ijps.v5n1p112

35. Ni C, Liu X, Hua Q, Lv A, Wang B, Yan Y. Relationship between coping, self- esteem, individual factors and mental health among Chinese nursing students: A matched case- control study. J Nurse Educa Today. 2010; 30(4): 338- 43.https://doi. org/10.1016/j.nedt.2009.09.003 PMID: 19800152

36. Luszczynska A, Sheng Cao D, Mallach N, Pietron K, Mazurkiewicz M, Schwarzer R. Intentions, planning, and self-efficacy predict physical activity in Chinese and Polish adolescents: Two moderated mediation analyses. Interna Jof Clin Heal \& Psych. 2010;10(2). 